\title{
ON HARDY'S INEQUALITY IN WEIGHTED REARRANGEMENT INVARIANT SPACES AND APPLICATIONS. II
}

\author{
LECH MALIGRANDA
}

\begin{abstract}
This note is a sequel to the preceding one with the same title published in these Proceedings. The contents of the first paper are assumed to be known. References are in alphabetical order in each paper, but they, as well as the theorems, are numbered consecutively.
\end{abstract}

We shall now apply our results to imbedding theorems for weighted Sobolev spaces and interpolation theorems.

1. Under the hypotheses of Theorems 1 or 2 , we have for every absolutely continuous function $x$ defined in $I=(0, l)$ such that

$$
x\left(0^{+}\right)=\lim _{t \rightarrow 0^{+}} x(t)=0, \quad \kappa_{1}(t) x^{\prime}(t) \in E,
$$

the following inequality holds:

$$
\|x\|_{E_{\kappa_{0}, t}} \leqslant C\left\|x^{\prime}\right\|_{E_{\kappa_{1}, r}},
$$

where $\kappa_{0}(t)=\kappa(t)[\psi(t)]^{-1}$ and $\kappa_{1}(t)=t \kappa(t)[\psi(t)]^{-1}$ or $\kappa(t)\left[\psi^{\prime}(t)\right]^{-1}$. Hence

$$
\|x\|_{E_{\kappa_{0}, t}} \leqslant C\left(\|x\|_{E_{\kappa_{1}, t}}+\left\|x^{\prime}\right\|_{E_{\kappa_{1}, t}}\right)=: C\|x\|_{W_{E, \kappa_{1}}^{1}} .
$$

2. A pair of quasi-Banach spaces $\left(X_{0}, X_{1}\right)$ continuously embedded in some Hausdorff topological vector space $\mathcal{X}$ is called a quasi-Banach couple. The sum of $X_{0}$ and $X_{1}$, denoted by $X_{0}+X_{1}$, is the set of all $x=x_{0}+x_{1}$, with $x_{i} \in X_{i}, i=0,1$. For each $x \in X_{0}+X_{1}$, we define the Peetre $K$-functional for $x$ by

$$
K(t, x) \equiv K\left(t, x ; X_{0}, X_{1}\right)=\inf _{\substack{x=x_{0}+x_{1} \\ x_{0} \in X_{0}, x_{1} \in X_{1}}}\left(\left\|x_{0}\right\|_{X_{0}}+t\left\|x_{1}\right\|_{X_{1}}\right) .
$$

The $K$ method generates interpolation spaces (see [22, Chapter 3]) by applying function quasi-norms $\Phi$ to $K(t, x)$. For example, if $0<p \leqslant \infty$ and $f: \mathbf{R}_{+} \rightarrow \mathbf{R}_{+}$is a continuous nondecreasing function such that $0<p^{\infty}(f) \leqslant q^{\infty}(f)<1$, then let us denote by $\left(X_{0}, X_{1}\right)_{f, p ; K}$ the set of all $x \in X_{0}+X_{1}$ for which

$$
\|x\|_{f, p ; K}=\left\{\int_{0}^{\infty}\left(\frac{K(t, x)}{f(t)}\right)^{p} d t / t\right\}^{1 / p}<\infty
$$

(see [24, p. 294]). In the sequel we write $\left(X_{0}, X_{1}\right)_{\theta, p ; K}$ instead of $\left(X_{0}, X_{1}\right)_{t^{\theta}, p ; K}$.

Received by the editors March 20, 1982.

1980 Mathematics Subject Classification. Primary 46E35, 46E30.

Key words and phrases. Interpolation spaces, rearrangement invariant spaces, indices. 
For $0<\theta<\theta^{\prime}<1$ and $x \in\left(X_{0}, X_{1}\right)_{\theta, 1 ; K}+\left(X_{0}, X_{1}\right)_{\theta^{\prime}, 1 ; K}$, we define

$$
\begin{aligned}
K_{\theta}^{\theta^{\prime}}(t, x) & \equiv K_{\theta}^{\theta^{\prime}}\left(t, x ; X_{0}, X_{1}\right) \\
& =K\left(t, a ;\left(X_{0}, X_{1}\right)_{\theta, 1 ; K},\left(X_{0}, X_{1}\right)_{\theta^{\prime}, 1 ; K}\right)
\end{aligned}
$$

and

$$
\begin{gathered}
\left(X_{0}, X_{1}\right)_{\Phi ; K_{\theta}^{\theta^{\prime}}}=\left\{x \in\left(X_{0}, X_{1}\right)_{\theta, 1 ; K}+\left(X_{0}, X_{1}\right)_{\theta^{\prime}, 1 ; K}:\right. \\
\left.\|x\|_{\Phi ; K_{\theta}^{\theta^{\prime}}}=\Phi\left(K_{\theta}^{\theta^{\prime}}(t, x)\right)<\infty\right\} .
\end{gathered}
$$

2a. We shall show that the space $E_{f, p}$, which by definition consists of all (class of) measurable functions on $(0,1)$ for which the norm

(30) $\|x\|_{f, p}=\int_{0}^{1} x^{*}(t) d t+\left\{\int_{0}^{1}\left[\frac{t}{f(1 /(1-\log t))} x^{* *}(t)\right]^{p} \frac{d t}{t(1-\log t)}\right\}^{1 / p}$

is finite, coincides with the interpolation space $\left(L^{1}, L \log ^{+} L\right)_{f, p ; K}$.

Theorem 3 (BenNeTt [2, TheOREM 8.1]-FOR $f(t)=t^{\theta}, 0<\theta<1$ ). Let $1 \leqslant p \leqslant \infty$ and $0<a \leqslant t f^{\prime}(t) / f(t) \leqslant b<1$. Then

$$
\left(L^{1}, L \log ^{+} L\right)_{f, p ; K}=E_{f, p}
$$

holds with equivalent norms.

Proof. As in [2] we have

$$
\begin{aligned}
\|x\|_{f, p ; K} \leqslant & 2\left\{\frac{\int_{0}^{e^{-1}}\left[(1-\log t) f(1 /(1-\log t))^{-1} \int_{t}^{1} x^{* *}(s) d s\right]^{p} d t}{t(1-\log t)}\right\}^{1 / p} \\
& +f\left(2^{-1}\right)^{-1}\left\{\int_{e^{-1}}^{1}\left[(-\log t)^{-1} \int_{t}^{1} x^{* *}(s) d s\right]^{p} e d t\right\}^{1 / p} \\
& +\left(\int_{1}^{\infty} f(t)^{-p} d t / t\right)^{1 / p} \int_{0}^{1} x^{*}(s) d s=I_{1}+I_{2}+I_{3} .
\end{aligned}
$$

We use the Hardy inequality (23) to obtain the estimate

$$
I_{1} \leqslant C\left\{\frac{\int_{0}^{1}\left[t f(1 /(1-\log t))^{-1} x^{* *}(t)\right]^{p} d t}{[t(1-\log t)]}\right\}^{1 / p}
$$

The rest of the proof is the same as in [2, Theorem 8.1] and need not be repeated here.

2b. We consider $L\left(\varphi_{i}, q_{i}\right), i=0,1$, spaces on $(0, \infty)$ (see Example 2). We assume that

$$
\left\{\begin{array}{l}
t^{-a} \varphi_{1}(t) \text { increases for some } a>0, \\
\eta(t)=\varphi_{0}(t) / \varphi_{1}(t) \text { is increasing from } 0 \text { to } \infty \\
t^{-b} \eta(t) \text { increases for some } b>0 .
\end{array}\right.
$$


TheOREM 4. Suppose that r.i. space $E$ on $(0, \infty)$ has Fatou norm or $\beta^{\infty}(E)<1$. Let $\varphi_{0}, \varphi_{1}$ be as given in above and let $t \tau^{\prime}(t) \approx \tau(t)(\tau$-function from Example 1$)$. If

$$
\Phi(h)=\left\|h\left(\eta(t)^{\theta^{\prime}-\theta}\right) / \varphi_{\theta}(t)\right\|_{E_{1, \tau-1},}, \quad \varphi_{\theta}(t)=\varphi_{0}(t)^{1-\theta} \varphi_{1}(t)^{\theta},
$$

and

$$
q^{\infty}\left(\varphi_{\theta^{\prime}} \circ \tau^{-1}\right)<\alpha^{\infty}(E) \leqslant \beta^{\infty}(E)<p^{\infty}\left(\varphi_{\theta} \circ \tau^{-1}\right)
$$

then

$$
\left(L\left(\varphi_{0}, q_{0}\right), L\left(\varphi_{1}, q_{1}\right)\right)_{\Phi ; K_{\theta}^{\theta^{*}}}=E_{1, \tau^{-1}}^{(*)}
$$

with equivalent quasi-norms.

Proof. From Theorem 2.1 of Holmstedt [26] (see also [22, p. 52]) and Theorem 4.2 of Torchinsky [32] it follows that

$$
\begin{aligned}
\varphi_{\theta}(t) x^{*}(t) & \leqslant C \int_{0}^{\eta(t)} u^{-\theta} \varphi_{0} \circ \eta^{-1}(u) x^{*} \circ \eta^{-1}(u) d u / u \\
& \leqslant C \int_{0}^{\eta(t)} u^{-\theta}\left[\sup _{0<s \leqslant \eta^{-1}(u)} \varphi_{0}(s) x^{*}(s)\right] d u / u \\
& \leqslant C \int_{0}^{\eta(t)} u^{-\theta}\left\|x^{*} \chi_{\left(0, \eta^{-1}(u)\right]}\right\|_{L\left(\varphi_{0}, q_{0}\right)} d u / u \\
& \leqslant C \int_{0}^{\eta(t)} u^{-\theta} K(u, x) d u / u \leqslant C K_{\theta}^{\theta^{\prime}}\left(\eta(t)^{\theta^{\prime}-\theta}, x\right)
\end{aligned}
$$

and

$$
\begin{aligned}
K_{\theta}^{\theta^{\prime}}\left(\eta(t)^{\theta^{\prime}-\theta}, x\right) \leqslant & C \int^{\eta(t)} u^{-\theta} K(u, x) d u / u+C \eta(t)^{\theta^{\prime}-\theta} \int_{\eta(t)}^{\infty} u^{-\theta^{\prime}} K(u, x) d u / u \\
\leqslant & C \int_{0}^{\eta(t)} u^{-\theta}\left\|x^{*} \chi_{\left(0, \eta^{-1}(u)\right]}\right\|_{L\left(\varphi_{0}, 1\right)} d u / u \\
& +C \int_{0}^{\eta(t)} u^{1-\theta}\left\|\varphi_{1} x^{*} \chi_{\left[\eta^{-1}(u), \infty\right)}\right\|_{L_{s}^{\prime}-1} d u / u \\
& +C \eta(t)^{\theta^{\prime}-\theta} \int_{\eta(t)}^{\infty} u^{-\theta^{\prime}}\left\|x^{*} \chi_{\left(0, \eta^{-1}(u)\right]}\right\|_{L\left(\varphi_{1}, 1\right)} d u / u \\
& +C \eta(t)^{\theta^{\prime}-\theta} \int_{\eta(t)}^{\infty} u^{1-\theta^{\prime}}\left\|\varphi_{1} x^{*} \chi_{\left[\eta^{-1}(u), \infty\right)}\right\|_{L_{s}^{1}-1} d u / u \\
\leqslant & C \varphi_{\theta}(t)\left\{\left[P_{\varphi_{\theta}}\left(P_{\varphi_{0}} x^{*}\right)\right](t)+\left[P_{\varphi_{\theta}}\left(Q_{\varphi_{1}} x^{*}\right)\right](t)\right. \\
& \left.+\left[Q_{\varphi_{\theta}}\left(P_{\varphi_{0}} x^{*}\right)\right](t)+\left[Q_{\varphi_{\theta}}\left(Q_{\varphi_{1}} x^{*}\right)\right](t)\right\} .
\end{aligned}
$$

Since (32) and $p\left(\varphi_{\theta}\right) \leqslant p\left(\varphi_{0}\right), q\left(\varphi_{1}\right) \leqslant q\left(\varphi_{\theta^{\prime}}\right)$ so by Theorem 1 (see Remark 4) we have

$$
\Phi\left(K_{\theta}^{\theta^{\prime}}(\cdot, x)\right) \approx\|x\|_{E_{\mid, \tau}^{*+1} \cdot 1} .
$$

In the particular case, $\varphi_{i}(t)=t^{1 / p_{i}}, 1 \leqslant p_{0}<p_{1}<\infty$, and $\tau(t)=t^{r\left(1 / p_{0}-1 / p_{1}\right)}$, $0<r<\infty$, Theorem 4 was obtained by Merucci [27, Theorem 1]. 
REMARK 7. If

$$
\begin{aligned}
& K_{\theta}^{1}(t, x)=K\left(t, x ;\left(X_{0}, X_{1}\right)_{\theta, 1: K}, X_{1}\right) \\
& K_{0}^{\theta^{\prime}}(t, x)=K\left(t, x ; X_{0},\left(X_{0}, X_{1}\right)_{\theta^{\prime}, 1: K}\right)
\end{aligned}
$$

and

$$
K_{0}^{1}(t, x)=K\left(t, x ; X_{0}, X_{1}\right)
$$

then Theorem 4 holds for $0 \leqslant \theta<\theta^{\prime} \leqslant 1$.

THEOREM 5. Suppose that r.i. space $E$ on $(0, \infty)$ has Fatou norm or $\beta^{\infty}(E)<1$. Let $\varphi_{i}, \psi_{i}, i=0,1, \eta(t)=\varphi_{0}(t) / \varphi_{1}(t), \xi(t)=\psi_{0}(t) / \psi_{1}(t)$ be as in $(31), 1 \leqslant q_{i}<r_{i} \leqslant \infty$, and let $t \tau^{\prime}(t) \approx \tau(t)(\tau$-function from Example 1$)$.

If $A \in\left[L\left(\varphi_{i}, q_{i}\right), L\left(\psi_{i}, r_{i}\right)\right], i=0,1$, and

$$
q^{\infty}\left(\varphi_{1} \circ \tau^{-1}\right)<\alpha^{\infty}(E) \leqslant \beta^{\infty}(E)<p^{\infty}\left(\varphi_{0} \circ \eta^{-1}\right),
$$

then $A \in\left[E_{1, \tau^{-1}}^{(*)}, E_{\kappa, \delta}^{(*)}\right]$, where $\delta(t)=\xi^{-1} \circ \eta \circ \tau^{-1}(t)$ and $\kappa(t)=$ $\psi_{0} \circ \delta(t) / \varphi_{0} \circ \tau^{-1}(t)$.

Proof. Let $\Phi(h)=\left\|h \circ \eta(t) / \varphi_{0}(t)\right\|_{E_{1, \tau}-1}$. Since

$$
\begin{aligned}
c \varphi_{0}(t) x^{*}(t) & \leqslant K\left(\eta(t), x ; L\left(\varphi_{0}, q_{0}\right), L\left(\varphi_{1}, q_{1}\right)\right) \\
& \leqslant C \varphi_{0}(t)\left[P_{\varphi_{0}} x^{*}(t)+Q_{\varphi_{1}} x^{*}(t)\right]
\end{aligned}
$$

and (34), so by Theorem 1 or Theorem 2 (see Remark 4)

$$
\left(L\left(\varphi_{0}, q_{0}\right), L\left(\varphi_{1}, q_{1}\right)\right)_{\Phi: K}=E_{1, \tau^{-1}}^{(*)} .
$$

On the other hand,

$$
\begin{aligned}
c \kappa(t) x^{*} \circ \delta(t) & \leqslant K\left(\eta \circ \tau^{-1}(t), x ; L\left(\psi_{0}, r_{0}\right), L\left(\psi_{1}, r_{1}\right)\right) / \varphi_{0} \circ \tau^{-1}(t) \\
& \leqslant C \kappa(t)\left[\left(P_{\psi_{0}} x^{*}\right)(\delta(t))+\left(Q_{\psi_{1}} x^{*}\right)(\delta(t))\right],
\end{aligned}
$$

and by Theorem 1,

$$
\left(L\left(\psi_{0}, r_{0}\right), L\left(\psi_{1}, r_{1}\right)\right)_{\Phi: K}=E_{\kappa, \delta}^{(*)} .
$$

From the interpolation theorem (see [ 22 or $\mathbf{2 7}$, Lemma 1]) we have thus proved our theorem.

Corollary 5. Let $\varphi_{i}, \psi_{i}, q_{i}, r_{i}, i=0,1, \eta, \xi$ be as in Theorem 5. Assume that $\tau(t)=t$ and $E=L(\varphi, q)$, where $q^{\infty}\left(\varphi_{1}\right)<p^{\infty}(\varphi) \leqslant q^{\infty}(\varphi)<p^{\infty}\left(\varphi_{0}\right)$. If $A \in$ $\left[L\left(\varphi_{i}, q_{i}\right), L\left(\psi_{i}, r_{i}\right)\right], i=0,1$, then $A \in[L(\varphi, q), L(\psi, q)]$, where $\psi(t) / \psi_{0}(t)=$ $\varphi \circ \eta^{-1} \circ \xi(t) / \varphi_{0} \circ \eta^{-1} \circ \xi(t)$.

REMARK 8. In the particular case, if $\varphi_{i}(t)=t^{1 / p_{i}}, 1 \leqslant p_{0}<p_{1}<\infty, \psi_{i}(t)=t^{1 / p_{i}^{\prime}}$, $1 \leqslant p_{0}^{\prime}<p_{1}^{\prime}<\infty$, then from Corollary 5 we have interpolation theorems of Sharpley [30], Bennett and Rudnick [21, Theorem B] and Persson [29, Theorem 2.1].

9. The reader will have no difficulty in supplying the proofs of Corollary 5 for the spaces $\Lambda(\varphi, q)$ because $\Lambda(\varphi, q)=L\left(\varphi^{1 / q}, q\right)$. 
10. The above theorems can be proved for Lorentz-Orlicz spaces $L(\varphi, F)$, $\Lambda(\varphi, F)$; some generalizations of Corollary 5 are in interesting papers of Torchinsky [31] and Heinig-Vaughan [25].

2c. In this part we extend the fundamental interpolation theorem of Krein and Semenov (see [10, Theorem 6.1, p. 175]) and the result of Pavlov [28, Theorem 3] to weighted r.i. spaces.

Theorem 6. Suppose that r.i. space $E$ on $\mathbf{R}_{+}$has Fatou norm or $\beta^{\infty}(E)<1$. Let $\varphi_{i}$, $\psi_{i}, i=0,1$, be positive nondecreasing concave functions on $\mathbf{R}_{+}$such that

(1) $\varphi_{0}(s) / \varphi_{1}(s)$ is nondecreasing, $\varphi_{0}\left(0^{+}\right)=0$;

(2) $\left\{\varphi_{0}(s) / \varphi_{1}(s): s \in \mathbf{R}_{+}\right\} \subset\left\{\psi_{0}(s) / \psi_{1}(s): s \in \mathbf{R}_{+}\right\}$.We assume that a linear operator $A: D_{A} \supset E_{\rho, t}^{(*)} \rightarrow L^{0}$, where $\rho$ is a positive measurable function on $\mathbf{R}_{+}$, satisfies

$$
\sup _{t>0} \psi_{i}(t)(A x)^{* *}(t) \leqslant C_{i} \int_{0}^{\infty} x^{*}(s) d \varphi_{i}(s), \quad i=0,1
$$

If

$$
q^{\infty}\left(\varphi_{1} / \rho\right)<\alpha^{\infty}(E) \leqslant \beta^{\infty}(E)<p^{\infty}\left(\varphi_{0} / \rho\right)
$$

then $A \in\left[E_{\rho, t}^{(*)}, E_{\kappa, \delta}^{(* *)}\right]$, where $\delta$ is a positive measurable solution of equation

$$
\psi_{0}(\delta(t))\left[\psi_{1}(\delta(t))\right]^{-1}=\varphi_{0}(t)\left[\varphi_{1}(t)\right]^{-1}
$$

and

$$
\kappa(t)=\psi_{0}(\delta(t))\left[\varphi_{0}(t)\right]^{-1} \rho(t)=\psi_{1}(\delta(t))\left[\varphi_{1}(t)\right]^{-1} \rho(t)
$$

Proof. We have (for $x \in \Lambda_{\varphi_{0}}+\Lambda_{\varphi_{1}}$ )

$$
(A x)^{* *}(\delta(t)) \leqslant C\left[\psi_{0}(\delta(t))^{-1} \int_{0}^{t} x^{*}(s) d \varphi_{0}(s)+\psi_{1}(\delta(t))^{-1} \int_{t}^{\infty} x^{*}(s) d \varphi_{1}(s)\right]
$$

(see [10, p. 176]). Hence,

$$
\begin{aligned}
\kappa(t)(A x)^{* *}(\delta(t)) & \leqslant C \rho(t)\left[S_{\varphi_{0}} x^{*}(t)+T_{\varphi_{1}} x^{*}(t)\right] \\
& \leqslant C \rho(t)\left[P_{\varphi_{0}} x^{*}(t)+Q_{\varphi_{1}} x^{*}(t)\right] .
\end{aligned}
$$

By applying Theorem 1 we have

$$
\|A x\|_{E_{\kappa, \delta}^{(* *)}} \leqslant C\left\|\rho P_{\varphi_{0}} x^{*}\right\|_{E}+C\left\|\rho Q_{\varphi_{1}} x^{*}\right\|_{E} \leqslant C\left\|\rho x^{*}\right\|_{E}=C\|x\|_{E_{\rho, l}^{(*)}} .
$$

Theorem 6 can be applied to generalizations of interpolation theorems, CalderonMitjagin [10, Theorem 6.4] and Marcinkiewicz [9, Theorem 1; 25, Theorem 5] or Hausdorff-Young theorem for convolution operator [10, Theorem $6.16 ; 28$, Theorem 4] which include weighted r.i. spaces as intermediate classes. The reader will have no difficulty in extending the results to the case of weighted r.i. spaces. 


\section{REFERENCES}

21. C. Bennett and K. Rudnick, On Lorentz-Zygmund spaces, Dissertationes Math. (Rozprawy Mat.) 175 (1979), 67 pp. MR 81i \#42020.

22. J. Bergh and J. Löfström, Interpolation spaces, an introduction, Springer-Verlag, New York, 1976. MR 58 \# 2349.

23. A. P. Calderon, Spaces between $L^{1}$ and $L^{\infty}$ and the theorem of Marcinkiewicz, Studia Math. 26 (1966), 273-299. MR 34 \#3295.

24. J. Gustavsson, A function parameter in connection with interpolation of Banach spaces, Math. Scand. 42 (1978), 289-305. MR 80d \#46124.

25. H. P. Heinig and D. Vaughan, Interpolation in Orlicz spaces involving weights, J. Math. Anal. Appl. 64 (1978), 79-95. MR 80f \#46028b.

26. T. Holmstedt, Interpolation of quasi-normed spaces, Math. Scand. 26 (1970), 177-199.

27. C. Merucci, Quelques resultats d'interpolation pour les espaces de Lorentz, C. R. Acad. Sci. Paris Sér. A 280 (1975), 1731-1734. MR 51 \#11085.

28. E. P. Pavlov, An interpolation theorem, Functional Analysis, No. 8: Operator Theory, Ul'janovsk. Gos. Ped. Inst. Ul'janovsk, 1977, pp. 113-119. (Russian) MR 58 \# 30149.

29. L. E. Persson, On a weak-type theorem with applications, Proc. London Math. Soc. 38 (1979), 295-308. MR 81b \#42062.

30. R. Sharpley, Spaces $\Lambda_{\alpha}(X)$ and interpolation, J. Funct. Anal. 11 (1972), 479-513. MR 49 \#611.

31. A. Torchinsky, Interpolation of operations and Orlicz classes, Studia Math. 59 (1976), 177-204. MR 55 \# 11024.

32. $\ldots$, The K-functional for rearrangement invariant spaces, Studia Math. 64 (1979), 175-190. MR 80k \#46032.

The Mathematical institute of the Polish Academy of Sciences, Poznań Branch, Mielżý́śiego 27/29, 61-725 Poznań, Poland 